\title{
A SPECTROSCOPIC STUDY OF $\eta$ CAR AND THE HOMUNCULUS
}

\author{
D. John Hillier \\ Joint Institute for Laboratory Astrophysics, University of Colorado \\ Boulder, Colorado 80309-0440, USA \\ David A. Allen \\ Anglo-Australian Observatory \\ PO Box 296, Epping, NSW 2121, Australia
}

Spectrophotometric maps of the peculiar massive star $\eta$ Car and its associated nebulae (the homunculus) were obtained in 1986. The spatial resolution is better than 1.5 arcseconds $(0.7$ arcseconds per pixel). Spectral coverage extends from $4000 \AA$ to $1 \mu \mathrm{m}$ at low ( $3 \AA / \mathrm{pixel})$ resolution, with additional observations of selected regions obtained at moderate resolution $(0.7 \AA / \mathrm{pixel})$.

We find dramatic differences in line profiles and line strengths across the homunculus. Both lobes show predominantly red shifted emission, with largest velocities occurring in the NW lobe. Our spectra confirm earlier suggestions that the spectrum of the homunculus arises predominantly through outflowing dust scattering light from the central object.

Variations in line ratios between spectra of the central object and homunculus force us to conclude that two distinct regions give rise to the majority of the observed emission lines. Light from the central object is viewed through a dusty disk which also gives rise to the [Fe II] lines. Because the continuum, and also the bulk of the $\mathrm{H}$ and $\mathrm{He} \mathrm{I}$ emission, arises inside the attenuating dust the [Fe II] lines appear strongest (and have the largest equivalent widths) on the central object. In the scattering lobes the continuum starlight is only weakly attenuated; therefore the scattered $[\mathrm{Fe} \mathrm{II}]$ emission is relatively weak.

Interpretation of the spectra is confused by the possible presence of overlying emission due to material not related to the homunculus - material which may be related to the outlying condensations. We see emission lines of CaII, [Ca II], Na I, [Cr II], [Fe II], and [Ni II] which may be intrinsic to the homunculus. Some of these features show peculiar velocities - we find blue shifted [CaII] emission offset to the NW $(\approx 2$ arcseconds $)$ from the central object.

A more detailed account of this work is in preparation. A recent introduction to the literature on $\eta$ Car can be found in the paper of Viotti et al. $(1990, A p$. J. Suppl., 71, 983). 